\title{
Mental health in elderly in the era of economic crisis in Greece.
}

\author{
Ilias Grammatikopoulos* \\ University of Ioannina, Greece
}

Accepted on October 14, 2017

The world's population is ageing rapidly. Between 2015 and 2050, the proportion of the world's older adults is estimated to almost double from about $12 \%$ to $22 \%$. Although older people make important contributions to society as family members, volunteers and as active participants in the workforce, they face special physical and mental health challenges which need to be recognized. The most common neuropsychiatric disorders in this age group are dementia and depression, while anxiety disorders affect $3.8 \%$ of the elderly population and over $20 \%$ of adults aged 60 and over suffer from a mental or neurological disorder [1].

As WHO stated "Mental health and emotional wellbeing are as important in older age as at any other time of life" , the interventions and mental disorders prevention actions are still poor, especially in countries that have been affected mostly from the economic crisis like Greece. From a national population survey of psychiatric morbidity in Greece presenting data of common mental disorders and use of services completed in 2010 (at the beginning of the economic crisis), from a representative sample of almost 5000 people aged $17-80$, the results revealed that $73,9 \%$ of participants with at least one common mental disorder (CMD) did not receive any treatment for their psychological health, 52\% have never spoken to the family physician and $73.3 \%$ has not spoken to a mental health professional in the past year [2].

Mental health problems are under-identified by healthcare professionals and older people themselves, and the stigma surrounding mental illness makes people reluctant to seek help [3], therefore mental disorders prevention strategies and accessible mental health services especially in Primary Care should be in the priority agenda.

Multiple risk factors are even more complicated in older people, due to the comorbidity of biological diseases with psychological factors and life stressors that determine the level of mental health of elderly people.

For example, older adults with physical health conditions such as heart disease have higher rates of depression than those who are medically well. Conversely, untreated depression in an older person with heart disease can negatively affect the outcome of the physical disease and prevalence of depression is important in older adults because the consequences include high costs of medical treatment, accentuation of both psychiatric and physical symptoms, difficulties with selfmanagement and adherence to medical treatment [1].
The treatment options should be holistic and be patientcentered including a multidisciplinary specialist service in old age psychiatry, providing continuity of care and establishing equity and quality of life. The mental health services should be integrated into the primary care network and social welfare system. A special concern should be addressed in training of negative professional attitudes towards older people with mental disorders, in order to reduce stigma and discrimination. The ageist notion that older people are inflexible and unable to change, reinforces popular and professional beliefs that mental disorders in this age group are incurable [4].

The economic crisis in Greece has influenced patients' adherence to therapy in rural and urban areas as well as their psychological and emotional status. Most common emotions reported were those of sadness, fear, stress, anxiety and isolation therefore is an urgent need for action within the context of primary care [5]. Therefore, in older people as a target group, interventions providing support in community and residential settings with the principles of occupational therapy and tailored physical activity programs could enhance mental disorders prevention and help them maintain or improve their mental wellbeing [6].

Establishing priorities especially in limited budget resources, as in Greece in the era of economic crisis, should focus in mental health promotion and mental disorders prevention interventions in particularly aged groups that are cost-effective. Training of all the existing mental health professionals in the special mental health problems of the elderly and establishment of multidisciplinary groups in primary health care centers should be in the priority of the Mental Health Policy agenda.

\section{References}

1. http://www.who.int/mediacentre/factsheets/fs381/en/

2. Skapinakis P, Bellos S, Koupidis S, et al. Prevalence and socio-demographic associations of common mental disorders in a nationally representative sample of the general population of Greece. BMC Psychiatry. 2013;13:163.

3. http://www.who.int/mental_health/media/en/499.pdf

4. Grammatikopoulos I. The geriatric giant: Mental health and well-being in elderly. Ment Health Fam Med. 2016;12:110-1. 
Citation: Grammatikopoulos I. Mental health in elderly in the era of economic crisis in Greece. J Ment Health Aging. 2017;1(1):10-11.

5. Tsiligianni I, Papadokostakis P, Prokopiadou D, et al. Impact of the financial crisis on adherence to treatment of a rural population in Crete, Greece. Qual Prim Care. 2014;22(5):238-44.

6. NICE Quideline. Mental wellbeing in over 65s: Occupational therapy and physical activity interventions. Public Health Guideline. 2008.

\section{*Correspondence to:}

Ilias Grammatikopoulos

University of Ioannina

Greece

E-mail: ilias17grams@yahoo.gr 